\title{
SONOGRAPHY OF THE LUNGS AND PLEURA
}

\author{
Afshan Pathan
}

\begin{abstract}
The role of Transthoracic Sonography in the evaluation of chest diseases has already been accepted throughout the world. Though Ultrasound (US) is not widely established as a routine method, but the advantages of low-cost, bedside availability and lack of ionizing radiation exposure has made it an indispensable diagnostic tool in the modern pulmonary medicine. US supplements the conventional chest X-ray and computed tomography and closes the gap between them. Besides, it is invaluable in the interventional procedures of chest and pleural space and it is suited to critically ill patients in need of bedside investigations. This article reviews the sonographic morphology of the pleural and peripheral pulmonary diseases.
\end{abstract}

KEY WORDS: Thoracic imaging. Pleural diseases. Pleural Neoplasm. Pulmonary lesions. Pulmonary malignancy.

\section{INTRODUCTION}

The ultrasound (US) waves are completely reflected by air space (such as in lung parenchyma) and bony thorax. This fact led to the mistaken notion that sonography is not a useful diagnostic tool in the diseases of chest. Despite this dissuasion, the first ultrasound examinations of the lung were performed 30 years ago. Ever since reports have been published regularly on pleuropulmonary sonographic diagnostic and therapeutic procedures. ${ }^{1,2}$ In recent years, Transthoracic ultrasound (TU) has received increased interest from radiologist and pulmonologist to evaluate a wide range of peripheral parenchymal, pleural and chest wall diseases. Modern ultrasound devices are lightweight and portable, which make them useful for bedside investigations and follow ups of the patients in intensive care unit. US is increasingly used to guide interventional procedures of the chest. Above all, it is cost effective in comparison to CT or conventional Xray. This article presents the review of the technique, clinical uses and sonographic findings in a variety of pleural, and pulmonary parenchymal abnormalities.

\section{Examination Technique and Instrumentation}

Before performing the US examination, it is a routine to review the patient's chest radiograph to localize the area of interest. The anterior and posterior chest is best imaged with the patient sitting upright, arms elevated and hands clasped behind the neck. ${ }^{3}$ The patient may also be scanned in the prone or supine positions. The lateral chest may be assessed in the lateral decubitus position. To optimize the imaging, caudal parts of the lung may be examined from subxiphoid and transdiaphragmatic views. ${ }^{6}$ Anterior and middle mediastinum is evaluated using a suprasternal ap- proach, performed with the patient in a supine position, shoulders supported with a pillow and head extended backward. ${ }^{4,5}$ Raising the arm above the patient's head increases the intercostals space to facilitate the scanning in both erect or recumbent positions. The optimal frequency of the transducer for Transthoracic US (TU) varies with the age of the patient, the location of the lesion, and the planned approach. However, a higher frequency (5- or 7.5-MHz) transducer provides better resolutions of near structures, such as the chest wall and pleura and a $3.5-\mathrm{MHz}$ transducer is more suitable for the visualization of deeper lesions. ${ }^{6}$ However, a convex array probe (3.5 to $5 \mathrm{MHz}$ ) combines the advantage of adequate close resolution and the ability to access deeper structures between the ribs. $^{3} \quad$ A linear or convex transducer usually has a broad view of the field and is better than a sector scanner for screening. However, for the lesions with a small US window or a very narrow intercostal space, a sector transducer is generally preferred. ${ }^{4,5,6,7}$ Color Doppler sonography can be used as a complementary method to provide additional information about the etiology of pleural and pleural-based pulmonary lesions diagnosed on the basis of radiography. This technique can simultaneously display blood flow information and Doppler spectral analysis. ${ }^{8}$

\section{SONO-ANATOMY OF CHEST WALL, PLEURA AND LUNG}

The sonography of normal chest wall shows multiple echogenic layers, representing the subcutaneous tissue, intercostal muscles and fascia. When scanned along the long axis, the margins of normal ribs are outlined as uninterrupted echogenicities. However, on transverse scans the ribs appear as curvilinear structures, casting posterior acoustic shadowing. ${ }^{5,7}$ 
A high-frequency linear US probe applied to an intercostal space enables visualization of the parietal and visceral pleura, as two thin bright echogenic lines ${ }^{9,10}$ measuring up to $2 \mathrm{~mm}$ thick (Figure 1). However, with a 3.5-MHz curvilinear probe, they loose differentiation and appear as echogenic bands. At real-time imaging, back-and-forth movements of the echogenic pleural line during respiration is referred as lung sliding sign. ${ }^{9,10}$

Inability of US beam to pass the air-filled lung prevents the visualization of normal lung parenchyma. However, due to large change in the acoustic impedance at pleura-lung interface, US images of the lung parenchyma display a pattern of two opposed types of reverberation artifacts. ${ }^{10,11}$ One, the horizontal artifacts which appear as a series of parallel echogenic lines equidistant from each other and other, the vertical (comet-tail) artifacts, seen to arise from last intercostals space above the diaphragm, spreading up to the edge of the screen (Figure I). The lung ultrasonography is possible when a pathologic process extends to the pleural surface, replaces the air within the lung, thus providing an acoustic window.

\section{FIGURE I:}

Normal US appearance of the chest. Below the relatively echogenic subcutaneous tissue, the intercostal muscles appear hypoechoic but contain mutiple echogenic fascia planes. The pleural interface appears as an echogenic line. The sharp change in the acoustic impedance at this interface results in reverberation artifacts (*), appearing as a series of horizontal lines parallel to the pleural interface. Vertical comet tail artifacts (+) can also be seen.

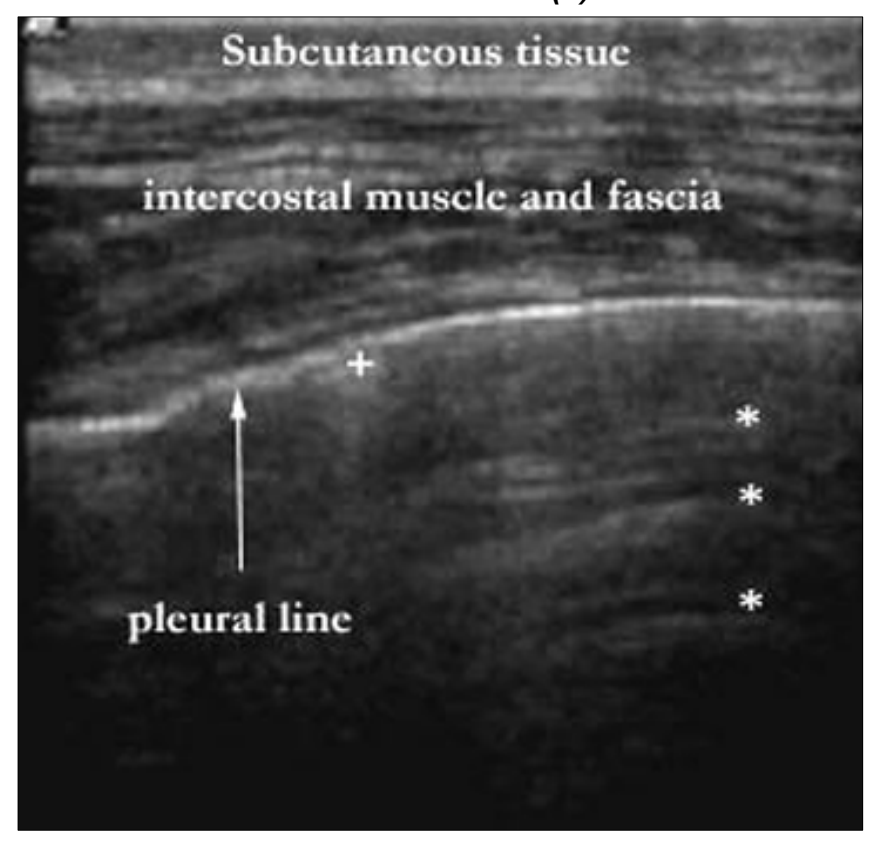

(Reprinted with permission from Reference 5)

\section{DISEASES OF PLEURA}

The indications and uses of chest US for pleural diseases are as follows: ${ }^{10}$

1- To detect pleural effusion, especially in minimal or loculated effusions and guide thoracocentesis and drainage.

2- To distinguish between fluid-filled and solid lesions in the presence of hemithorax opacification on chest radiograph.

3- To differentiate subpulmonary effusion from subphrenic fluid accumulation and diaphragm paralysis in radiographically elevated hemidiaphragms.

4- To assess the invasion of tumors to the pleura and chest wall, and guide the transthoracic needle biopsy of the tumors.

5- To clarify the nature of unknown pleural densities and guide needle biopsy of the pleura.

6- To recognize pneumothorax, especially for emergency situations, or when roentgenography equipment is not readily available.

\section{Pleural Effusions}

Real-time ultrasound evaluation of pleural collections accurately determines the size and nature of the effusion and may be useful in predicting the success of thoracentesis. We can obtain sonographic evidence of even $20 \mathrm{ml}$ of pleural effusion accurately in comparison to 50-70 ml on chest x-ray. Pleural effusion appears as an echo-free or dark zone between the visceral and parietal portions of the pleura. ${ }^{10}$ The thoracic US is particularly useful in the intensive care unit setting where small effusion or the real amount of an effusion may be missed, especially when radiographs are made in a supine position, or when radiograms are shot with a sub optimal radiographic technique. ${ }^{10,}$ 12,13 Though Ultrasound is sensitive but it is less specific for detecting minimal effusion. RG Wu et al 14 have observed that minimal or loculated (even 3 to 5 $\mathrm{ml}$ ) effusions are readily detected by demonstrating "fluid color" sign by Doppler US with the sensitivity of $89.2 \%$ and specificity of $100 \%{ }^{14,15}$ The sign refers to the presence of color signal within the fluid collection, believed to arise from transmitted respiratory and cardiac movements.

At US transudate is always anechoic. While exudates may appear anechoic or complex, with small moving dots, fronds, septations or loculations. ${ }^{16,17}$ The sonographic evidence of thickened pleura (more than 3 $\mathrm{mm}$ ) is also highly suggestive of exudates. ${ }^{16}$ When pleural collection becomes homogenously echogenic, mimicking a solid mass, it represents an empyma ${ }^{5}$ (Figure II). It has been reported that Ultrasound guided percutaneous aspiration is usually successful in effusions that are anechoic or complex with movable septae. ${ }^{16,17}$ But it is less likely to be successful in 
the pleural effusion with fixed septae, loculations or an echogenic pleural collection. ${ }^{17}$

FIGURE II:

In this patient with an empyema, note the diffusely echogenic pleural collection

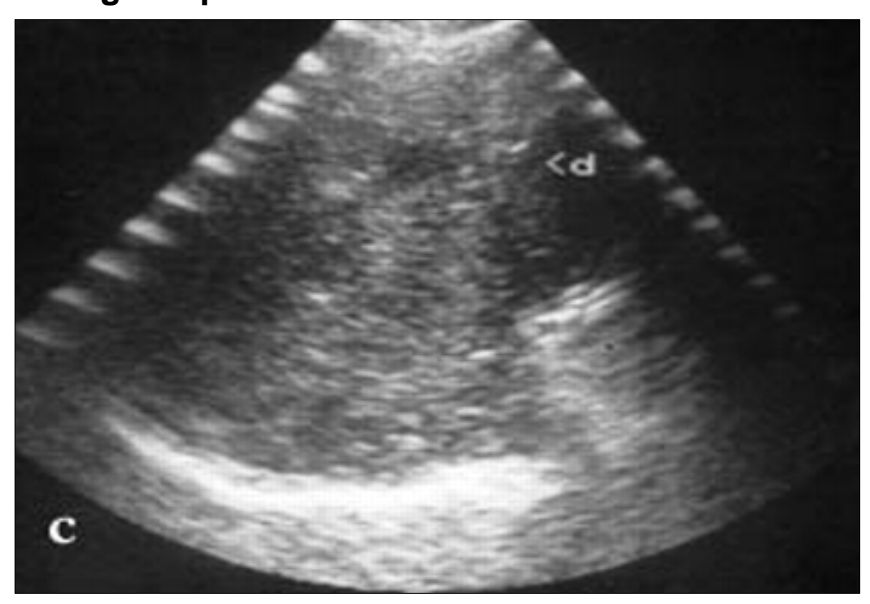

(Reprinted with permission from Reference 5)

\section{Quantification of Pleural Effusions}

Several formulae exist for sonographic quantifying of pleural effusions. A useful technique for clinical purposes is to obtain the transverse section in supine position, as even intensive care unit patients can be examined in this position. Furthermore, in the supine position free pleural fluid collects predominantly dorsally and consistently manifests as a sickle-shaped lamella on transverse sonographic scans. The maximum thickness of the fluid layer, which represents the total effusion volume, is determined by measuring the perpendicular distance between the posterior chest wall and the posterior margin of the lung. ${ }^{18}$ With this method, an effusion width of $20 \mathrm{~mm}$ had a mean volume of $380 \mathrm{~mL} \pm 130$ (standard deviation), while one of $40 \mathrm{~mm}$ had a mean volume of $1,000 \mathrm{~mL} \pm 330$. The prediction error with sonographic measurement (mean, $224 \mathrm{~mL}$ ) was statistically significantly less than that with radiographic measurement (mean, $465 \mathrm{~mL}){ }^{18}$

\section{Pneumothorax and Hydropneumothorax}

Pneumothoraces are usually detected by means of a combination of clinical examination and chest radiography. A subtle pneumothorax may be overlooked in a supine anteroposterior radiograph in the trauma patients. Besides, radiographs obtained in these patients are difficult to interpret due to sub optimal technical factors, artifacts and widespread lung changes which can mask or mimic pneumothorax. ${ }^{19,20}$ Therefore several groups of investigators ${ }^{11,19,21}$ have found TS a cost-effective and safe bed-side-method, for an immediate exclusion or diagnosis of pneumothorax, in the emergency bedside patients. The sonographic signs used to diagnose pneumothorax include ${ }^{11,20,21}$ absent lung sliding sign, loss of vertical (comet-tail) reverberation artifacts and augmented horizontal reverberation artifacts. The false-positive results for pneumothorax may occur in patients with emphysema, pleural adhesions or subcutaneous emphysema, where lung sliding sign may be absent with no pneumothorax. ${ }^{20} \mathrm{~A}$ potential pitfall may also occur in patients with large lung contusions or pleural calcifications, which results in acoustic artifacts, thus limiting the visualization of the pleural interface. ${ }^{19}$

Sonography appears to be a new approach to diagnose Hydropneumothorax (HPN), which is particularly useful during or after ultrasonically guided procedures including pleural drainage. HPN can also be identified with US through the panel of sonographic features including $^{22,23}$ the presence of the horizontal line (the air-fluid level), the absence of the lung sliding sign above the air-fluid level, the movement of the air-fluid level synchronously with respiration (the curtain sign) and the hyperechoic reflectors within the pleural effusion.

\section{Pleural Thickening}

US demonstrates pleural thickening as focal hypoechoic broadening of the pleura with or without irregular margins, just superficial to the echogenic pleural-lung interface ${ }^{5,9}$ (Figure III). Pleural thickening and adhesion are usually caused by pleuritis, empyema, hemothorax, or iatrogenic pleurodesis. Calcification may be detected with old tuberculosis. Small pleural effusion and pleural thickening both can appear anechoic on grayscale US. But on Doppler US, unlike pleural effusion, pleural thickening does not exhibit the fluid color sign because it has no movable parts. ${ }^{14,15}$ At US pleuritis appears as an interruption of the pleural line and irregular hypoechoeic thickening of the visceral pleura. ${ }^{24}$

\section{FIGURE III:}

US demonstrates pleural thickening as a hypoechoic band, just superficial to the echogenic pleural-lung interface

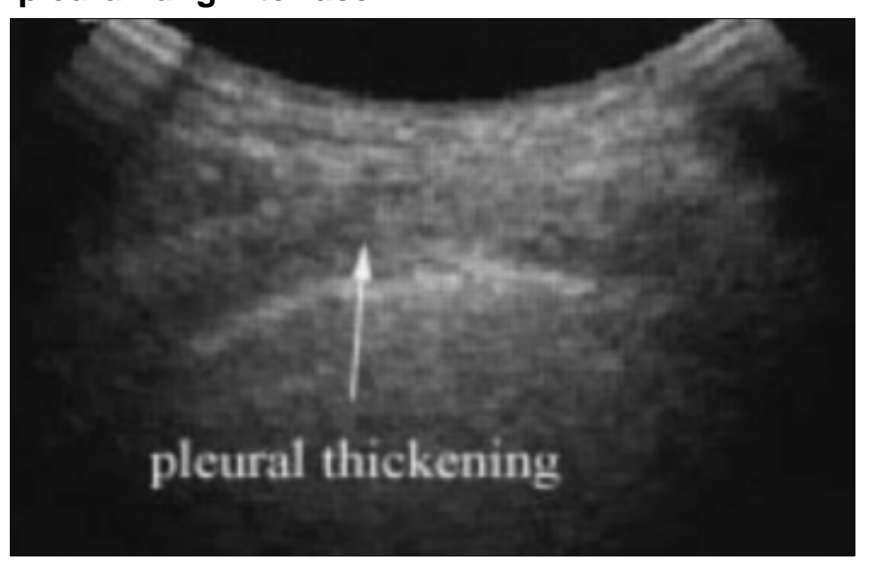

(Reprinted with permission from Reference 5) 


\section{Pleural Tumors}

Pleural tumors are rare, except for malignant pleural mesothelioma. At US mesothelioma appears as diffuse or irregular nodular thickening of the pleura. ${ }^{1,25}$ US-guided core needle biopsy is very helpful for pathologic differentiation between pleural fibrosis and pleural tumor. However, CT remains the modality of choice in the diagnosis and preoperative staging of malignant mesothelioma. ${ }^{26}$ The echogenic and welldemarcated ${ }^{1,26}$ metastatic nodules may be detected on the parietal and diaphragmatic pleura. The combined findings of pleural fluid and nodules, or sheetlike diffuse thickening, in a patient with a known primary malignancy, are highly suggestive of metastatic disease. $^{26}$

\section{Pulmonary Parenchymal Lesions Pulmonary Consolidation}

US is able to demonstrate pleural-based consolidation. Consolidation appears heterogeneous with ventilation and homogeneous when lung is airless, resembling the echo-texture of the liver. ${ }^{3,27}$ Multiple bright dot like and branching linear structures are seen with in the consolidation. These findings, termed as sonographic air bronchogram (Figure IV), represent air in the patent bronchi and scattered residual air in alveoli. $^{28,29,30}$ The fluid bronchograms, the anechoic tubular structures, representing fluid-filled airways due to bronchial obstruction, may also be observed in consolidation. $^{31}$ The size of the pneumonia appears smaller at US than on radiographs. Because the artifacts from the well-aerated lung produce hazy images, thus limiting complete visualization of the lesion. ${ }^{3,31}$

Although pneumonia and tuberculosis are the common causes of lung consolidation, infarction, hemorrhage and bronchoalveolar carcinoma can also result in consolidation that appears similar at US. ${ }^{3}$ In that case repeated examinations usually show changes with inflammatory diseases, whereas malignant lesions do not change their pattern within days. Moreover, benign acute disorders present with a different clinical picture. ${ }^{3}$ When the diagnosis is uncertain, US may be used to guide lung biopsy. Pneumonia and tuberculosis can undergo necrosis leading to lung abscess formation. Identification of hypoechoic lesion with arc or ring-shaped, well-defined or irregular wall favors the lung abscess. ${ }^{1}$ The center of the abscess is usually anechoic butmay contain internal echoes and septations.
FIGURE IV:

US demonstrates an area of consolidation within the right lower lobe. The texture of the consolidated lung appears isoechoic to the liver. Multiple echogenic foci are seen within the consolidated lung and correspond to air-filled airways

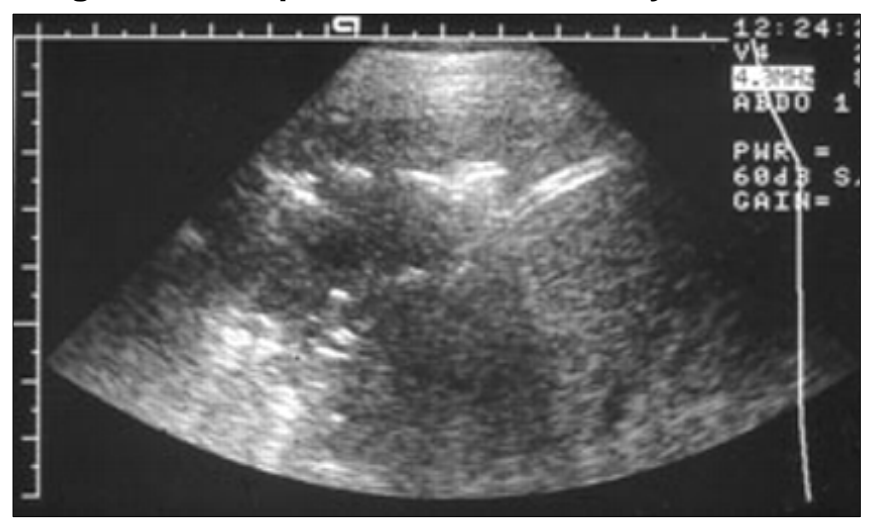

(Reprinted with permission from Reference 5)

\section{Atelectasis}

On ultrasound the passive atelectasis appears as a hyperechoic, homogeneously structured organ with a smooth surface. It floats in the anechoic pleural fluid synchronous with respiration ${ }^{10}$ (Figure V). Due to overall decrease in lung volume the bronchi, if not obstructed, can be seen as dot-like echogenic structures, crowded together in very close apposition in one plane. $^{32}$ The rounded atelectasis has been described as a pleural based hypoechoic mass, associated with thickening of the adjacent pleural and extrapleural fat. An echogenic line, representing the scarred, invaginated pleura, can be seen extending from the pleura into the mass in $86 \%$ of cases. ${ }^{33}$

FIGURE V:

A large anechoic effusion with passive atelectasis of the underlying lung is seen

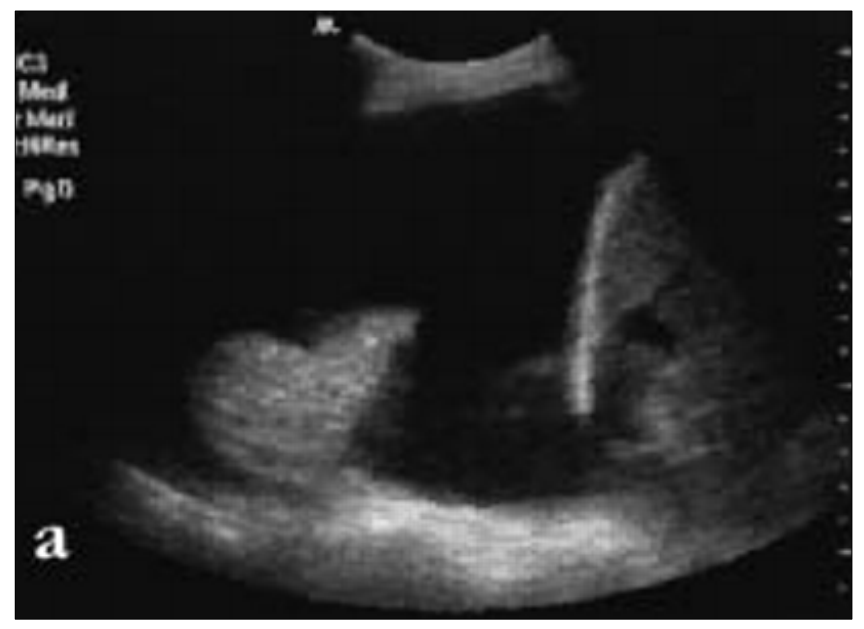

(Reprinted with permission from Reference 5) 


\section{Congenital Cystic Lung Diseases}

Congenital cystic lung diseases demonstrated at US include pulmonary sequestration and cystic adenomatoid malformation. Having similar US findings they both appear as a complex echogenic mass, with a cystic or homogeneously solid appearance. ${ }^{34,35}$ Color flow imaging may also be helpful to differentiate between the two. When echogenic pulmonary mass with or without cysts is discovered, particularly in the lower lobes, use of a subxiphoid approach to search for an anomalous vessel arising from the aorta may be helpful to differentiate pulmonary sequestration from cystic adenomatoid malformation. ${ }^{35}$

\section{Primary Lung Carcinoma}

US is an additional tool in the diagnosis of lung tumor. The Peripheral malignant lung tumor appears as a hypoechoic or echogenic mass. ${ }^{36}$ Consolidation with fluid bronchograms may be seen adjacent to the lung tumor which is associated with obstructive pneumonitis. ${ }^{31,37}$ Sometimes the obstructing tumor is seen as a hyperechoic mass inside the partially consolidated or collapsed lung. ${ }^{38}$ The Pulmonary blastoma, a childhood tumor, presents as a solitary complex echogenic mass, occupying an entire lobe or hemithorax ${ }^{6}$ On the other hand, the central malignant tumors may be visualized, if accompanied by atelectasis or obstructive pneumonia of the affected lobe. ${ }^{3}$

As mentioned above, the malignant lung tumor associated with obstructive pneumonitis ${ }^{37}$ may present as consolidations, so the main decision-making about peripheral consolidation is differentiating between benign and malignant disease ${ }^{3}$. The lung consolidated by malignancy has a distorted and tuberous surface. It moves stiffly and does not change its shape during respiration. However, benign consolidations shows smooth surface. On the other hand, benign and malignant lung lesions may be differentiated by their vascular pattern and blood flow using color Doppler US. ${ }^{8,39}$ But the procedure has limitations, as some pulmonary vessels are too small for blood flow to be detected. However, Color Doppler signal may be obtained in peripheral malignant masses in a significant proportion (64\%) of cases. ${ }^{39}$ Malignant masses are associated with the presence of distorted tortuous vessels and neovascularity, ${ }^{28,29}$ showing low-impedance flow in comparison to benign tumor which usually had relatively high impedance flow. ${ }^{39} \mathrm{WH}$ Hsu et al reported that constant flow pattern is representative of the true neovascularity of lung cancers, whereas a pulsatile or triphasic flow pattern may be seen in both benign and malignant neoplasms. ${ }^{4}$ It has been shown that a lower pulsatility index, resistive index, and peak systolic velocity but a higher end diastolic velocity are characteristics of malignancy. ${ }^{39,40}$

\section{Metastasis}

CT scanning/ spiral CT scanning is the most accurate imaging modality to detect small nodules of the lung. However, in a patient with a history of underlying malignancy, one or more nodules detected at US, may represent pulmonary metastasis. They usually appear as multiple subpleural hypoechoic nodules ${ }^{7}$ or echogenic well-demarcated lesions measuring about 1-2 $\mathrm{cm}$ in diameter. Color Doppler imaging demonstrates the high vascularity and high blood flow of these lesions. ${ }^{41}$

\section{REFERENCES}

1. Mathis G. Thoraxsonography. I. Chest and pleura. Ultrasound Med Biol. 1997; 23:1131-1139.

2. Tidwell AS. Ultrasonography of the thorax (excluding the heart). Vet Clin North Am Small Anim Pract. 1998; 28(4):993-1015.

3. Sonja Beckh, Pál L. Bölcskei, et al. Real-Time Chest Ultrasonography. Chest. 2002;122:17591773.

4. Krejci CS, Trent EJ, Dubinsky T. Thoracic sonography. Respir Care. 2001; 46(9):932-9.

5. Dow-Mu Koh, Burke S, Davies N, et al. Transthoracic US of the Chest: Clinical Uses and Applications. Radiographics. 2002;22:e1.

6. Kim OH, Kim WS, Kim MJ, et al. US in the Diagnosis of Pediatric Chest Diseases. Radiographics. 2000;20:653-671.

7. Diacon $\mathrm{AH}$, Theron J, Bolliger CT. Transthoracic ultrasound for the pulmonologist. Curr Opin Pulm Med. 2005;11(4):307-12

8. Görg C, Seifart U, Görg K, et al. Color Doppler Sonographic Mapping of Pulmonary Lesions. J Ultrasound Med. 2003; 22:1033-1039.

9. Wernecke K. Ultrasound study of the pleura. Eur Radiol.2000; 10(10):1515-23.

10. Tsai TH, Yang PC. Ultrasound in the Diagnosis and Management of Pleural Disease. Curr Opin Pulm Med. 2003; 9(4):282-290.

11. Lichtenstein D, Meziere G, Biderman P, et al. The comet-tail artifact: an ultrasound sign ruling out pneumothorax. Intensive Care Med. 1999; 25 $(4): 3838$.

12. Laing FC, Filly RA, et al. Problems in the application of ultrasonography for the evaluation of pleural opacities. Eur J Radiol. 2005; 53(3):463-70.

13. Kocijancic I, Vidmar K, Ivanovi-Herceg Z. Chest sonography versus lateral decubitus radiography in the diagnosis of small pleural effusions. J Clin Ultrasound. 2003; 31(2):69-74.

14. Wu RG, Yang PC, Kuo SH, et al. Fluid color sign: a useful indicator for discrimination between pleural thickening and pleural effusion. J Ultrasound Med. 1995; 14:767-769. 
15. Yang P. Applications of colour Doppler ultrasound in the diagnosis of chest diseases. Respirology.1997 Sep;2(3):231-8.

16. Sajadieh H, Afzali F, Sajadiesh V, et al Ultrasound as an alternative to aspiration for determining the nature of pleural effusion, especially in older people. Ann N Y Acad Sci. 2004;1019:585-92.

17. Yang PC, Luh KT, Chang DB, et al. Value of sonography in determining the nature of pleural effusion: analysis of 320 cases. Am J Roentgenol. 1992; 159:29-33.

18. Klemens L. Eibenberger, WoLfgang I. Dock, Markus E. Ammann, et al 'Quantification of Pleural Effusions: Sonography versus radiography' Radiology. 1994; 191(3):681-4.

19. Chan SS. Emergency bedside ultrasound to detect pneumothorax. Acad Emerg Med. 2003; 10 (1):91-4.

20. Goodman TR, Traill ZC, Phillips AJ, et al. Ultrasound detection of pneumothorax. Clin Radiol. 1999; 54:736-739.

21. Lichtenstein DA, Meziere G, Lascols $\mathrm{N}$ et al. Ultrasound diagnosis of occult pneumothorax. Crit Care Med. 2005; 33(6):1425-6

22. Reissig A, Kroegel C. Accuracy of transthoracic sonography in excluding post-interventional pneumothorax and hydropneumothorax. Comparison to chest radiography. Eur J Radiol. 2005; 53 (3):463-70.

23. Targhetta R, Bourgeois JM, Chavagneux R, et al. Ultrasonographic approach to diagnosing hydropneumothorax. Chest. 1992; 101: 931-934.

24. Gehmacher O, Kopf A, Scheier M, et al. Can pleurisy be detected with ultrasound?. Ultraschall Med. 1999; 18:214-219.

25. Herth F. Diagnosis and staging of mesothelioma transthoracic ultrasound. Lung Cancer. 2004; 45 Suppl 1:S63-7.

26. Steinberg, HV, Erwin, BC. Metastases to the pleura: sonographic detection. J Clin Ultrasound. 1987;15,276-279.

27. Targhetta R, Chavagneux R, Bourgeois JM, et al. Sonographic approach to diagnosing pulmonary consolidation. Ultrasound Med Biol. 1995.

28. Acunas B, Celik L, Acunas A. Chest sonography: differentiation of pulmonary consolidation from pleural disease. Acta Radiol. 1989; 30:273-275.

29. Gorg C, Seifart U, Holzinger I, et al. Bronchioloalveolar carcinoma: sonographic pattern of 'pneumonia'. Eur J Ultrasound 2002;15(3):109-17.

30. Weinberg B, Diakoumakis EE, Kass EG, et al. The air bronchogram: sonographic demonstration. AJR Am J Roentgenol 1986;147,593-595.

31. Dorne $\mathrm{HI}$, et al. Differentiation of pulmonary parenchymal consolidation from pleural isease using the sonographic fluid bronchogram. Radiology. 1986; 158(1):41-2.

32. Baysal K, Uysal S, Cetinkaya F, et al. Twodimensional ultrasonographic findings of atelectatic lung segments. Indian J Pediatr 1997; 64 (5):713-7.

33. Marchbank MD, Wilson AG, Joseph AE. Ultrasound features of folded lung. Clin Radiol. 1996; 51:433-437.

34. Rosado-de-Christenson ML, Stocker JT. Congenital cystic adenomatoid malformation. RadioGraphics $1991 ; 11: 865-886$.

35. West MS, Donaldson JS, Shkolnik A. Pulmonary sequestration: diagnosis by ultrasound. J Ultrasound Med. 1989; 8:125-129.

36. Ko JC, Yang PC, Luh KT, et al. Lobar bronchioloalveolar carcinoma: an ultrasound study. J Formos Med Ass. 1992; 91(11):1049-53.40.

37. Yang PC, Luh KT, Wu HD, et al. Lung tumors associated with obstructive pneumonitis: US studies. Radiology. 1990; 174(3 Pt 1):717-20.2pa

38. Hsu WH, Chiang CD, Hsu JY, Huang WL. Detection of mass lesions in the collapsed lung by ultrasonography. J Formos Med Assoc 1992; 91(1): 57-62.

39. Yuan A, Chang DB, Yu CJ, et al. Color Doppler sonography of benign and pulmonary masses. Am J Roentgenol 1994; 163:545-549.

40. WH Hsu, Ikezoe J, Chen CY, et al. Color Doppler ultrasound signals of thoracic lesions: correlation with resected histological specimens. Am J Resp Crit Care Med. 1996; 153:1938-1951.

41. Liae YS, Yang PC, Yuan A. Ultrasonography and color Doppler imaging of metastatic pulmonary choriocarcinoma. Chest. 1993; 104:1600-1601.

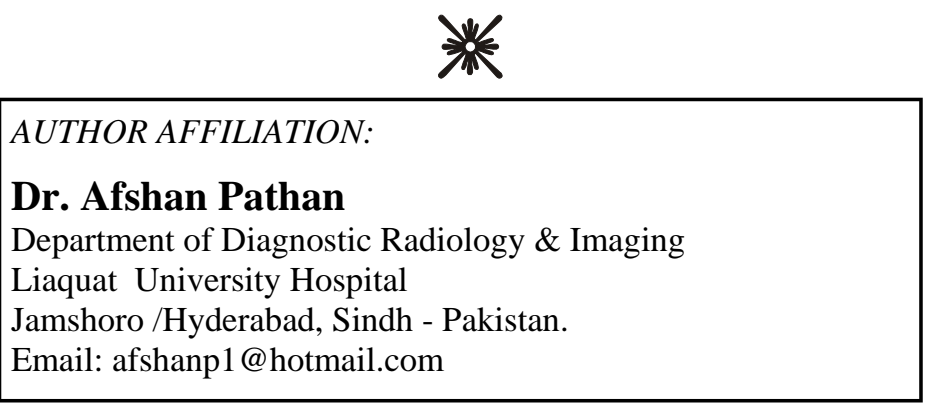

